\title{
Gestão da assistência farmacêutica em municípios do estado da Paraíba (PB): olhando a aplicação de recursos públicos
}

\author{
Suelma de Fátima Bruns \\ Departamento de Ciências Farmacêuticas, Centro de Ciências da Saúde, \\ Universidade Federal da Paraíba (DCF/CCS/UFPB) \\ Vera Lucia Luiza \\ Núcleo de Assistência Farmacêutica, Escola Nacional de Saúde Pública Sergio Arouca, Fundação Oswaldo \\ Cruz (NAF/ENSP/Fiocruz) \\ Egléubia Andrade de Oliveira \\ Instituto de Estudos em Saúde Coletiva, Área de Bioética \\ Universidade Federal do Rio de Janeiro (UFRJ)
}

\begin{abstract}
No contexto da gestão pública contemporânea, este estudo teve por objetivo verificar o desempenho de distintos aspectos da assistência farmacêutica em municípios da Paraíba. Trata-se de uma pesquisa descritiva, realizada a partir de relatórios de fiscalização dos municípios (no período de 2003 a 2010), produzidos pela Controladoria-Geral da União (CGU). O estudo apontou que 98,1\% dos municípios apresentaram pelo menos um problema na gestão de recursos e/ou de serviços farmacêuticos; que em $52,7 \%$ o controle de estoque não existia ou era deficiente; que em $52,7 \%$ não foram observadas as normas de aquisição de medicamentos; que em $38,2 \%$ foram constatados desvios de recursos e fraudes do programa; e que em $27,3 \%$ dos municípios faltavam medicamentos básicos. Conclui-se que houve importantes problemas de gestão que afetaram processos e a qualidade da prestação dos serviços aos cidadãos.
\end{abstract}

Palavras-chave: gestão; recurso público; política pública; assistência farmacêutica.

Gestión de servicios farmacéuticos en municipalidades del estado de Paraíba (PB): mirando a la aplicación de recursos públicos

En el contexto de la gestión pública contemporánea este trabajo tuve como objetivo verificar el disempeño de diferentes aspectos de los servicios farmacéuticos en municipalidades del estado de Paraíba. Tratase de un estudio descriptivo, a partir de los informes de inspección en las municipalidades (de 2003 a 2010), producidos por la Controladoria Geral da União (CGU). Si encontró que $98.1 \%$ de las municipalidades tienen al menos un problema en la gestión de recursos y/o servicios farmacéuticos;

DOI: http://dx.doi.org/10.1590/0034-76121502

Artigo recebido em 13 mar. 2013 e aceito em 5 fev. 2014.

Rev. Adm. Pública - Rio de Janeiro 48(3):745-765, maio/jun. 2014 
que en $52.7 \%$ el control de inventario no existía o era deficiente así como no fueron observadas la aplicación de las reglas de adquisición de medicamentos; que en $38.2 \%$ se encontraron desvío de recursos y fraude; y que en $27.3 \%$ de las municipalidades hay falta de medicamentos básicos. Si concluyó que había problemas de gestión que afectan a importantes procesos así como a la calidad de la prestación de servicios a los ciudadanos.

Palabras clave: gestión; recurso público; política pública; asistencia farmacéutica.

Management of pharmaceutical service in towns of Paraíba, Brazil: looking at the application of public resources

In the context of contemporary public management, this study aimed to check the performance of various aspects of pharmaceutical service in towns of Paraíba, Brazil. This is a descriptive research, conducted by means of inspection reports from the towns (within the period from 2003 to 2010), produced by the Office of the Inspector General of the Union (CGU). The study has found that $98.1 \%$ of the towns had at least one problem in the management of resources and/or pharmaceutical services; that in $52.7 \%$ stock control did not exist or it was deficient; that in $52.7 \%$ the rules for acquiring medicines were not observed; that in $38.2 \%$ misappropriation of resources and fraud concerning the program were found; and that in $27.3 \%$ of towns there was lack of basic medicines. It was concluded that there were important management problems that affected processes and the quality of services provided to citizens.

KEYWORDs: management; public resource; public policy; pharmaceutical service.

\section{Introdução}

A complexidade e velocidade das mudanças no mundo globalizado reforçam a importância fundamental do desenvolvimento e fortalecimento da gestão para sustentabilidade das organizações. Analisando a administração como um fenômeno universal do mundo moderno, Chiavenato (2003) sinaliza que empresas e organizações, com suas especificidades, necessitam empreender de forma coordenada uma gama de atividades, que envolvem desde a condução de pessoas e suas potenciais aptidões e a avaliação de desempenhos dirigidos a objetivos estabelecidos, até a alocação de recursos de maneira efetiva.

Nesse contexto, a administração pública tem como foco o gerenciamento dos resultados, buscando eficiência e eficácia administrativas, redução das desigualdades sociais, redução dos gastos e prestação de serviços com qualidade à população, tendo como fundamentos a ética, a moral e a jurisdição, princípios essenciais que devem ser assumidos por todos aqueles que administram os bens públicos em favor do cidadão e da justiça social.

Segundo Vasconcelos (2009), a eficiência está calcada em dois princípios, ambos com o mesmo objetivo, de alcançar os melhores resultados na prestação de serviço com menor dispêndio dos recursos públicos. Portanto, o primeiro está relacionado com a forma de atuação do agente público, resultando na premissa que desempenhe suas atribuições da melhor forma possível, e o segundo diz respeito ao modo de organizar, estruturar e disciplinar a administração pública. 
Nesse sentido, cabe assinalar a importância do controle externo constitucionalmente previsto, como o papel exercido pelo Tribunal de Contas da União (TCU). O TCU avalia não só a legalidade dos atos administrativos relacionados àqueles que implicam despesas, mas também os relacionados à eficiência, economicidade e legitimidade de tais atos. De forma semelhante, a Controladoria-Geral da União (CGU) é o órgão responsável, no âmbito do Poder Executivo, pelas atividades de auditoria pública, controle interno, correção, prevenção e combate à corrupção. Os relatórios de auditoria dos referidos órgãos fornecem subsídios para um diagnóstico do desempenho da administração pública, bem como para a avaliação da utilização de recursos públicos.

Num contexto onde tanto o aumento dos gastos com saúde quanto a demanda são crescentes, e os recursos são finitos para garantir uma assistência integral à saúde dos cidadãos, a assistência farmacêutica, em especial, tem merecido crescente atenção por parte de governantes, instituições e profissionais de todo o mundo. Segundo a Organização Mundial da Saúde (OMS), cerca de 2 bilhões de pessoas não têm acesso aos medicamentos essenciais, e estima-se que apenas $15 \%$ da população mundial consome mais do que $90 \%$ da produção farmacêutica (WHO, 2004).

Segundo o Conselho Regional de Medicina do Estado de São Paulo (Cremesp), “o Brasil ocupa o 9ำ lugar no mundo em termos de consumo de medicamentos, movimentando aproximadamente R $\$ 10$ bilhões de dólares por ano" (Cremesp, 2006:7). É importante ressaltar que vem sendo crescente o financiamento governamental para medicamentos (Vieira, 2009). Entretanto, uma parcela importante das pessoas obtém seus medicamentos por compra no mercado privado, por vezes após tentativa frustrada de obtê-los no setor público, inclusive medicamentos contidos na Relação Nacional de Medicamentos (Rename) (Boing, Bertoldi e Peres, 2011). O fenômeno da judicialização denuncia outro aspecto do problema, quando os cidadãos recorrem à via judicial para obtenção de seus tratamentos (Bruns, Luiza e Oliveira, 2013).

Portanto, a assistência farmacêutica se constitui hoje em um problema de saúde pública e um desafio para consolidação do Sistema Único de Saúde (SUS). Em situações de escassez de recursos, a gestão dos processos envolvidos é condição sine qua non na área farmacêutica, pois possibilita uma maior disponibilidade e, consequentemente, uma maior acessibilidade dos medicamentos pela comunidade (Ali, 2009).

São ainda poucos, apesar de crescentes, os estudos sobre o conhecimento do desempenho desses serviços de saúde, sobretudo em nível nacional.

Pesquisa realizada pela Organização Pan-Americana da Saúde (Opas), em parceria como o Ministério da Saúde (Opas, 2005), apontou para deficiências na gestão, nos mecanismos de financiamento e na integração das ações de assistência farmacêutica às ações de saúde, deixando clara a necessidade de investir para qualificação dos serviços.

Outro estudo realizado em nível nacional foi o de Vieira (2008), no qual foram utilizados os relatórios das auditorias da CGU, no período compreendido entre agosto de 2004 e julho de 2006, cujo foco era a qualificação dos serviços farmacêuticos no Brasil. 
A importância desses estudos reside na consolidação de modelos sistemáticos que podem ser aplicados para avaliação do desempenho da assistência farmacêutica, como forma de subsidiar as decisões na busca da qualificação dos serviços ofertados à população.

Faz-se, portanto, relevante ampliar os estudos sobre a problemática da gestão da assistência farmacêutica no âmbito do SUS, e, em termos práticos, ressaltar a importância de consolidar metodologias que possam ser úteis na análise dos dados disponíveis e no suporte à gestão das políticas públicas. Pois, apesar de ampla base de dados existente no país, persistem dificuldades para a realização de estudos sistemáticos com metodologias já consagradas para avaliação do desempenho da assistência farmacêutica.

Nesse sentido, as informações gerenciais desse setor são relevantes, tanto para a formulação e implementação da política de assistência farmacêutica, como para auxiliar os gestores na tomada de decisões, uma vez que refletem a realidade dos serviços por meio de indicadores seletivos e específicos, propiciando melhorias e racionalização da aplicação dos recursos (Brasil, 2011).

O presente estudo teve como objetivo analisar a utilização de recursos públicos destinados à Assistência Farmacêutica Básica de municípios da Paraíba e verificar o desempenho de distintos aspectos da assistência farmacêutica municipal, a partir de relatórios de fiscalização dos municípios (período de 2003 a 2010), produzidos pela CGU.

\section{O Sistema Único de Saúde e a assistência farmacêutica: avanços e desafios}

A assistência farmacêutica passou, desde 1998, com a publicação da Política Nacional de Medicamentos, a integrar formalmente a agenda de governo, com o compromisso da garantia da segurança, eficácia e qualidade dos medicamentos ao menor custo possível, da promoção do seu uso racional e o do acesso da população aos medicamentos essenciais. Foi também estabelecida a responsabilidade dos três entes federados - União, estados, Distrito Federal e municípios - e a gestão e o financiamento compartilhados (Brasil, 1998).

No entanto, a descentralização da assistência farmacêutica, ao mesmo tempo que promoveu o fortalecimento dos princípios do SUS, trouxe desafios aos gestores estaduais e municipais na promoção das mudanças para o novo modelo de gestão voltada ao cidadão. A efetivação da descentralização impôs como necessário o aperfeiçoamento e a busca de novas estratégias por parte dos gestores, com o intuito de promover a eficiência de suas ações e a consolidação e integralização dos vínculos entre os serviços e a população (Brasil, 1990).

Nesse sentido, para dar andamento ao processo de descentralização e buscando organizar de forma crescente a responsabilidade das esferas federativas na gestão da assistência farmacêutica, foi instituído o Incentivo à Assistência Farmacêutica na Atenção Básica (Iafab), e, através da Portaria no 176 , de 1999, foram estabelecidos os critérios e os requisitos para a habilitação dos estados e municípios a receberem esse incentivo financeiro do governo federal (Brasil, 1999). Sucedeu-se uma série de outras normativas ao longo do tempo, mantendo-se, porém, o caráter tripartite da gestão e do financiamento. 
Em 2004, foi publicada a Política Nacional de Assistência Farmacêutica, como parte integrante da Política Nacional de Saúde, que redefine a assistência farmacêutica como um "conjunto de ações voltadas à promoção, proteção e recuperação da saúde, tanto no nível individual como coletivo, tendo o medicamento como insumo essencial e visando ao acesso e ao seu uso racional". Essa política explicita a necessidade de qualificação dos serviços de assistência farmacêutica nos diversos níveis de atenção, o acesso e a promoção do uso racional de medicamentos como seus principais eixos estratégicos para melhoria da qualidade de vida da população (Brasil, 2004).

Entretanto, apesar dos avanços das políticas nacionais de medicamentos e assistência farmacêutica - uma vez que elas representam importantes referenciais para a reorientação e qualificação dos serviços de assistência farmacêutica —, muitos problemas perduram, comprometendo a missão de garantir o acesso da população aos medicamentos e seu uso racional (Vieira, 2010). Nesse sentido, estudos demonstram dificuldades de acesso da população aos medicamentos essenciais, o que se reflete em prejuízo à saúde do cidadão (Opas, 2005; Oliveira, Bermudez e Osorio-de-Castro, 2007; Siqueira e Gaudard, 2009).

A gestão da assistência farmacêutica se constitui em um dos maiores desafios de governo, na área da saúde, devido ao seu alto custo e grau de complexidade, que envolve aspectos assistenciais, técnicos, logísticos e tecnológicos, aspectos culturais, políticos, econômicos e sociais, bem como baixa qualificação dos serviços farmacêuticos, falhas nos componentes constitutivos do Ciclo da Assistência Farmacêutica, aumento crescente da demanda de medicamentos pela população, escassez e má gestão dos recursos públicos.

A implementação de uma logística adequada pode ter impactos positivos em termos de acesso e qualidade dos serviços prestados, refletindo-se na melhoria de eficiência e de controle de gastos no serviço público (Vaz e Lotta, 2011).

Desse modo, indubitavelmente, uma gestão eficiente da política de assistência farmacêutica é considerada estratégica para otimizar os escassos recursos destinados à saúde.

A assistência farmacêutica depende decisivamente de operações logísticas para a sua execução, envolvendo todo o Ciclo da Assistência Farmacêutica, que é constituído pelos componentes organizacionais: programação, aquisição, armazenamento e dispensação de medicamentos, permeados por mecanismos de gestão, aos quais podemos chamar de governança. Entretanto, esse ciclo tem sua interface pautada nas ações da atenção à saúde com foco no usuário, transcendendo as questões puramente logísticas.

Segundo o Banco Mundial (2007), "governança é a forma pela qual o poder é exercido na administração dos recursos, sociais e econômicos de um país visando o desenvolvimento, e a capacidade dos governos de planejar, formular e programar políticas e cumprir funções". Na saúde, a governança também se reporta à maneira pela qual a organização, seus gestores e seus funcionários respondem por seus comportamentos (ex.: gestão de recursos, planejamento, monitoramento de serviços, gestão financeira etc.) na prestação de serviços com qualidade e eficiência, bem como na prestação de contas (Banco Mundial, 2007). Segundo Marin e colaboradores (2003), o componente governança tem sido adotado como princípio norteador da assistência farmacêutica no SUS. 
Torna-se, portanto, imprescindível que os governos, nos três níveis de gestão, priorizem a questão do planejamento, execução e gestão dos recursos alocados para a assistência farmacêutica, com transparência e responsabilidade, garantindo o accountabiliy, em conformidade com os princípios do SUS e com amparo legal.

\section{Procedimentos metodológicos}

Trata-se de um estudo descritivo, a partir de base de dados secundários obtidos dos relatórios de fiscalização de municípios quanto à utilização de recursos públicos, produzidos pela Controladoria-Geral da União (CGU), órgão responsável, no âmbito do Poder Executivo, pelas atividades de auditoria pública, controle interno, correção, prevenção e combate à corrupção. Baseia-se na metodologia utilizada por Vieira (2008), que abordou o país como um todo.

Para avaliar a execução dos orçamentos da União, fiscalizar a implementação dos programas de governo e fazer auditorias e fiscalizações sobre a gestão dos recursos públicos federais sob a responsabilidade de órgãos e entidades públicos, foi criado, em abril de 2003, o Programa de Fiscalização a partir de Sorteios Públicos, que usa o mesmo sistema de sorteio das loterias da Caixa Econômica Federal para definir, de forma isenta, municípios e estados a serem fiscalizados quanto à utilização dos recursos públicos federais (Brasil, 2003).

Com os resultados dos sorteios, uma equipe da CGU se desloca até os municípios sorteados e procede in loco à auditoria, seguindo parâmetros previamente definidos para a verificação de toda a documentação pertinente, bem como para a averiguação das condições em que os serviços vêm sendo prestados à população. Em caso de constatação de qualquer irregularidade, é fornecido ao gestor responsável o direito de defesa e do contraditório, para que ele possa apresentar contraprovas e justificativas pertinentes. Ao término das auditorias, os relatórios concluídos são disponibilizados, na íntegra, na página eletrônica da CGU.

O estado da Paraíba, escolhido para este estudo, é uma das 27 unidades federativas do Brasil. Localizado na região Nordeste, possui uma extensão territorial de 56 mil km², distribuídos entre 223 municípios, com uma população de 3,77 milhões de habitantes (IBGE, 2010).

Para esta pesquisa, foram obtidos os dados, disponíveis no site da CGU, de todos os mu-

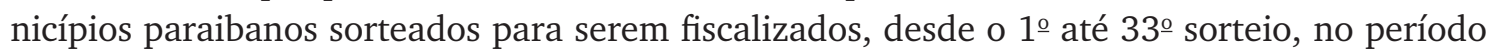
compreendido entre 2003 e 2010. Cumpre assinalar que os mesmos não foram contemplados no $1^{\circ}$ sorteio realizado em 2003 , aparecendo somente a partir do $2^{\circ}$ sorteio, também realizado em 2003. Dessa forma, a CGU realizou 73 fiscalizações em 68 municípios, uma vez que cinco municípios (Pitimbu [2003 e 2008], Araruna [2007 e 2010], Bom Sucesso [2003 e 2007], Caldas Brandão [2006 e 2010] e Várzea [2003 e 2004]) foram repetidos de acordo com os sorteios realizados.

A categorização das variáveis de interesse foi feita a partir dos problemas constatados pelos auditores, conforme frequência apresentada nos relatórios, e que representaram as prin- 
cipais dificuldades identificadas pelos mesmos quanto à Assistência Farmacêutica Básica nos municípios. Assim, foram criadas 16 categorias segundo os componentes do Ciclo da Assistência Farmacêutica abordados nas auditorias do TCU (quadro 1).

\section{Quadro 1}

\section{Categorias de análise segundo o componente do Ciclo da Assistência Farmacêutica}

\begin{tabular}{|c|c|}
\hline Componente & Categoria \\
\hline Programação & Ausência de programação e/ou procedimentos de controle \\
\hline \multirow[t]{4}{*}{ Aquisição } & Inobservância das normas de aquisição de medicamentos/processo licitatório irregular \\
\hline & Fracionamento de despesas para aquisição de medicamentos \\
\hline & $\begin{array}{l}\text { Aquisição de medicamentos com preço superior ao do fornecedor ou do banco de preços em } \\
\text { saúde (BPS) }\end{array}$ \\
\hline & Aquisição de medicamentos sem licitação \\
\hline \multirow[t]{3}{*}{ Armazenamento } & Controle de estoque deficiente/inexistente de medicamentos \\
\hline & Condições inadequadas de armazenamento \\
\hline & Medicamentos com validade expirada \\
\hline Dispensação & Falta de controle na dispensação de medicamentos \\
\hline \multirow[t]{6}{*}{ Governança } & Ausência do farmacêutico (responsável pelo Serviço de Assistência Farmacêutica) \\
\hline & Desvio de recursos e fraudes do programa de Assistência Farmacêutica \\
\hline & Falta de comprovação dos gastos com os recursos da Assistência Farmacêutica \\
\hline & Falta de contrapartida da Secretaria Estadual de Saúde (SES) \\
\hline & Falta de contrapartida da Secretaria Municipal de Saúde (SMS) \\
\hline & Inexistência de Plano Municipal de Assistência Farmacêutica Básica \\
\hline Resultado & Falta de medicamentos à população/Descumprimento do estoque mínimo e obrigatório \\
\hline
\end{tabular}

Fonte: Elaboração própria (2011).

Os dados foram sistematizados em planilhas do Excel® e, em seguida, exportados para um banco de dados do PostgreSQL, ${ }^{1}$ onde foram feitas a consolidação e a tabulação. As tabelas geradas foram novamente exportadas para o Excel, onde foram feitas as formatações.

\footnotetext{
${ }^{1}$ O PostgreSQL é um SGBD (Sistema Gerenciador de Banco de Dados), objeto relacional de código aberto, com mais de 15 anos de desenvolvimento. É extremamente robusto e confiável, além de ser extremamente flexível e rico em recursos.
} 


\section{Resultados e discussão}

A leitura e análise dos 73 relatórios das auditorias da CGU mostraram que a Parte Variável do Piso da Atenção Básica (PAB) para a Assistência Farmacêutica Básica constava de 55 relatórios de fiscalização $(75,3 \%)$ de 52 municípios, no período de 19 de maio de 2003 a 30 de setembro de 2010, sendo esta, portanto, a população objeto deste estudo (tabela 1).

\section{Tabela 1}

Frequência de municípios paraibanos com Relatórios de Assistência Farmacêutica por municípios sorteados, entre 2003 e 2010

\begin{tabular}{|lccc|}
\hline Ano & $\begin{array}{c}\text { Municípios paraibanos sorteados com } \\
\text { relatório da Controladoria-Geral da União }\end{array}$ & $\begin{array}{c}\text { Municípios paraibanos com relatório de } \\
\text { Assistência Farmacêutica }\end{array}$ & $\%$ \\
\hline 2003 & 11 & 11 & 100,0 \\
2004 & 14 & 10 & 71,4 \\
2005 & 10 & 4 & 40,0 \\
2006 & 6 & 6 & 100,0 \\
2007 & 9 & 7 & 77,8 \\
2008 & 6 & 5 & 83,3 \\
2009 & 9 & 5 & 55,6 \\
2010 & 8 & 7 & 87,5 \\
TOTAL & 73 & 55 & 75,3 \\
\hline
\end{tabular}

Fonte: Elaboração própria, com base nos relatórios de fiscalização da Controladoria-Geral da União (CGU), recuperados em: <www.cgu. gov.br/sorteios/index2.asp>. Acesso em: 17 maio 2011.

Assim, 23,3\% dos 223 municípios do estado puderam ser analisados, o que pode ser considerado uma abrangência satisfatória.

Quanto às constatações de irregularidades/falhas detectadas na assistência farmacêutica, $98,1 \%$ dos municípios paraibanos apresentaram problemas em pelo menos uma das categorias (figura 1 e tabela 2). Bigdeli e colaboradores (2012) mencionam a baixa responsabilidade e transparência no setor público como sérios desafios para a promoção do adequado acesso aos medicamentos nos países de renda baixa e média.

O elevado percentual de municípios que apresentaram problemas quanto à gestão da assistência farmacêutica reflete as dificuldades enfrentadas pelo SUS em prestar uma assistência farmacêutica de qualidade. De acordo com os relatos da auditoria da CGU, apenas 1,9\% dos municípios analisados no presente estudo não apresentaram problemas na condução da assistência farmacêutica.

Na tabela 2 encontram-se demonstrados os resultados obtidos concernentes aos componentes do Ciclo da Assistência Farmacêutica e sua frequência, segundo as categorias analisadas. 
Figura 1

\section{Frequência das categorias/problemas na Assistência Farmacêutica constatados nos relatórios da CGU em municípios paraibanos, entre 2003 e 2010}

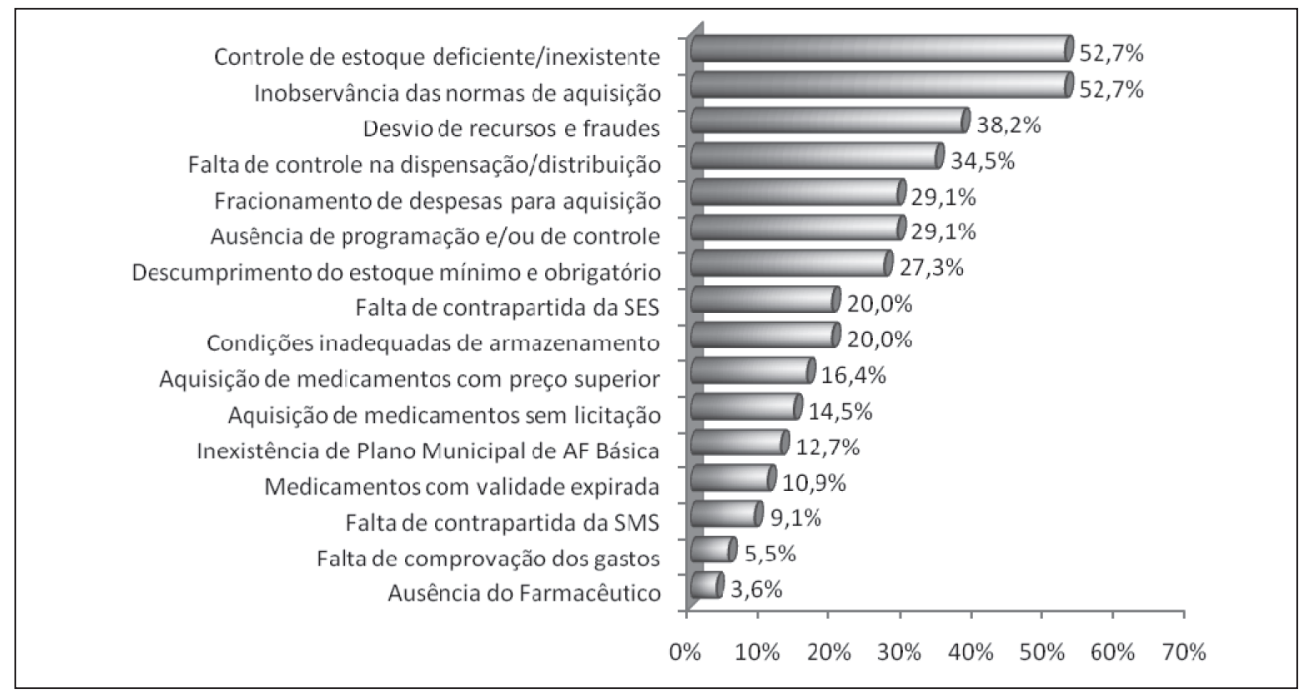

Fonte: Elaboração própria, com base nos relatórios de fiscalização da Controladoria-Geral da União (CGU), recuperados em: <www.cgu.gov.br/sorteios/index2.asp>. Acesso em: 17 maio 2011.

Tabela 2

\section{Frequência das categorias/problemas na Assistência Farmacêutica constatados nos relatórios da CGU em municípios paraibanos, entre 2003 e 2010}

\begin{tabular}{|c|c|c|c|}
\hline Componente & Categoria & Quantidade & $\%$ \\
\hline Programação & Ausência de programação e/ou procedimentos de controle & 16 & 29,1 \\
\hline \multirow[t]{4}{*}{ Aquisição } & $\begin{array}{l}\text { Inobservância das normas de aquisição de medicamentos/ } \\
\text { processo licitatório irregular }\end{array}$ & 29 & 52,7 \\
\hline & Fracionamento de despesas para aquisição de medicamentos & 16 & 29,1 \\
\hline & $\begin{array}{l}\text { Aquisição de medicamentos com preço superior ao do } \\
\text { fornecedor ou do banco de preços em saúde (BPS) }\end{array}$ & 9 & 16,4 \\
\hline & Aquisição de medicamentos sem licitação & 8 & 14,5 \\
\hline \multirow[t]{3}{*}{ Armazenamento } & Controle de estoque deficiente/inexistente de medicamentos & 29 & 52,7 \\
\hline & Condições inadequadas de armazenamento & 11 & 20,0 \\
\hline & Medicamentos com validade expirada & 6 & 10,9 \\
\hline Dispensação & Falta de controle na dispensação de medicamentos & 19 & 34,5 \\
\hline \multirow[t]{2}{*}{ Governança } & $\begin{array}{l}\text { Ausência do farmacêutico (responsável pelo Serviço de } \\
\text { Assistência Farmacêutica) }\end{array}$ & 2 & 3,6 \\
\hline & $\begin{array}{l}\text { Desvio de recursos e fraudes do programa de Assistência } \\
\text { Farmacêutica }\end{array}$ & 21 & 38,2 \\
\hline
\end{tabular}




\begin{tabular}{|llcc|}
\hline Componente & \multicolumn{1}{c}{ Categoria } & Quantidade & $\%$ \\
\hline & Falta de comprovação dos gastos com os recursos da & 3 & 5,4 \\
& Assistência Farmacêutica & 11 & 20,0 \\
& Falta de contrapartida da Secretaria Estadual de Saúde (SES) & 5 & 9,1 \\
& Falta de contrapartida da Secretaria Municipal de Saúde (SMS) & 7 & 12,7 \\
& Inexistência de Plano Municipal de Assistência Farmacêutica & & 27,3 \\
& Básica & 15 & \\
Resultado & Falta de medicamentos à população/Descumprimento do & & \\
& estoque mínimo e obrigatório &
\end{tabular}

Fonte: Elaboração própria, com base nos relatórios de fiscalização da Controladoria-Geral da União (CGU), recuperados em: <www.cgu. gov.br/sorteios/index2.asp>. Acesso em: 17 maio 2011.

\section{Componente - programação}

Constatou-se que, em 29,1\% dos municípios analisados, havia "ausência de programação e/ ou procedimentos de controle". Tal quadro é preocupante, pois as deficiências neste componente de gestão podem concorrer para aumentar os riscos de desperdício e de desabastecimento, comprometendo todos os demais componentes do Ciclo da Assistência Farmacêutica, resultando em sérios transtornos à população, pela falta de acesso aos medicamentos de que precisa.

O objetivo principal da programação é definir os quantitativos dos medicamentos selecionados que devem ser adquiridos, levando-se em consideração o perfil de doenças da população e as metas de cobertura e oferta de serviços. Deve-se observar, também, a modalidade de compra adotada, os níveis de estoque existentes, a capacidade de armazenamento, a disponibilidade do fornecedor e os recursos orçamentários e financeiros disponíveis. Uma programação eficaz garante a disponibilidade dos medicamentos nas quantidades adequadas e no tempo oportuno para atender à demanda da população, com base na lista oficial de medicamentos básicos.

\section{Componente - aquisição}

Em 52,7\% dos municípios paraibanos, foi detectada a "inobservância das normas de aquisição de medicamentos", o que constitui uma das principais falhas ou irregularidades encontradas nas licitações públicas. Além disso, em 29,1\% dos municípios, constatou-se o "fracionamento de despesas para aquisição de medicamentos", que se caracteriza por dividir a despesa estimada visando realizar a contratação direta ou utilizar modalidade de licitação menos complexa do que a prevista pela lei. Esse fato foi ratificado pelos auditores da CGU, quando analisadas as notas fiscais emitidas a favor de empresas, totalizando um montante financeiro muito acima do valor estipulado para dispensa de licitação (art. 24, II — limite R\$ 8.000,00), infringindo, portanto, o disposto no art. 23, § 5으, da Lei no 8.666, de 1993 (Brasil, 1993). 
Em uma situação de escassez de medicamentos, o controle sobre o preço possibilita uma maior disponibilidade e, consequentemente, uma maior acessibilidade aos mesmos pela comunidade (Carasso et al., 2009).

Em 16,36\% dos municípios, a "aquisição de medicamentos ocorreu com preço superior ao do fornecedor ou ao banco de preços em saúde", ${ }^{2}$ situação que caracteriza, no mínimo, a ausência de realização de pesquisa de preços para definição do valor unitário máximo a ser contratado pelo município para a aquisição dos medicamentos.

A atitude supracitada contraria as disposições do art. 15, inciso V, o qual faz a seguinte referência: sempre que possível, a gestão deve balizar-se pelos preços praticados no âmbito dos órgãos e entidades da Administração Pública; no art. 40, § 2º inciso II, da Lei no 8.666, de 1993, e no orçamento estimado em planilhas de quantitativos e preços unitários (Brasil, 1993). Os dados mostraram, ainda, que, em 14,5\% dos municípios estudados, a "aquisição de medicamentos ocorreu sem o processo licitatório."

O contexto acima descrito pode ser considerado de grande gravidade, pois caracteriza a transgressão de formalidades previstas na Lei no 8.666, de 1993 (Brasil, 1993), e na Lei no 10.520, de 2002 (Brasil, 2002). Essas infrações podem estar associadas ao despreparo das Comissões de Licitação, responsáveis pela condução das aquisições, ou, por vezes, vinculadas a fraudes.

Conforme o art. $3^{\circ}$ da Lei no $\mathbf{8} .666$, de 1993, o procedimento licitatório tem por objetivo garantir o princípio constitucional da isonomia e selecionar a proposta mais vantajosa para a administração pública, estando esta em plena conformidade com os princípios básicos da legalidade, da impessoalidade, da moralidade, da publicidade e da probidade administrativa. Um dos princípios consagrados de forma implícita é o da "economicidade", ao mencionar-se que o procedimento licitatório visa selecionar a proposta mais vantajosa para a administração pública.

Profissionalizar a gestão é fundamental, para evitar o superfaturamento, a corrupção, os procedimentos de aquisição ilegais e licitações que não seguem a legislação vigente, práticas que causam muitos prejuízos à sociedade (Benevides e Menezes, 2011).

\section{Componente - armazenamento}

Com base na análise realizada, constatou-se que 52,7\% dos municípios apresentaram um "controle de estoque deficiente ou inexistente". O resultado encontrado é preocupante, em virtude da importância de se dispor de um eficiente sistema de controle de estoque (entradas e saídas) para subsidiar a programação e a aquisição de medicamentos, corrigir distorções,

\footnotetext{
${ }^{2}$ O Banco de Preços em Saúde (BPS) é um sistema informatizado que registra, armazena e disponibiliza, por meio da internet, os preços de medicamentos e produtos para a saúde, que são adquiridos por instituições públicas e privadas cadastradas no sistema. Além de tornar públicas as informações, o sistema proporciona a visualização de relatórios gerenciais, visando auxiliar as instituições na gestão de seus recursos financeiros.
} 
evitar perdas, superposições ou desabastecimento, equilibrando, assim, a demanda e a oferta de medicamentos. De acordo com as recomendações de Fleury, Wanke e Figueiredo (2000), o controle de estoque deve especificar claramente o quanto pedir, quando pedir, quanto manter em estoques de segurança e onde localizar. Essas definições são importantes para subsidiar uma política de estoque, em resposta a cada uma dessas questões que envolvem diversas análises, tais como: o valor agregado dos produtos e a capacidade de projeção da demanda em conformidade às exigências dos consumidores finais, considerando prazos de entrega e disponibilidade do produto.

Existem várias formas para que se possa realizar o controle de estoque: de forma manual, através de fichas de prateleiras, de formulários, relatórios de acompanhamento, por meio de sistema informatizado, entre outros. Para que se possa ter confiabilidade e maior segurança das informações, deve-se adotar um duplo controle no seu acompanhamento (Marin et al., 2003).

$\mathrm{O}$ armazenamento consiste no "conjunto de procedimentos técnicos e administrativos que tem por finalidade assegurar as condições adequadas de conservação dos produtos" (Brasil, 2006). Para a realização de um armazenamento adequado, faz-se necessária a observância de alguns procedimentos, tais como: recebimento e conferência dos medicamentos, estocagem de acordo com as especificações dos produtos (termolábeis, fotossensíveis, inflamáveis), disposição conforme orientação do fabricante, sistema de organização que facilite a localização e o acesso rápido e fácil, preservação da integridade e qualidade dos medicamentos, assim como dispor de uma boa infraestrutura.

Foram constatados problemas relacionados às condições de armazenamento dos medicamentos nos municípios. Foi verificado que $20 \%$ dos municípios paraibanos incluídos no estudo apresentaram "condições inadequadas de armazenamento". Isso sugere que os medicamentos podem estar expostos a alterações na qualidade, perda e possíveis desvios.

Esse dado corrobora os encontrados no Rio Grande do Sul por De Bernardi, Bieberbach e Thomé (2006), onde um percentual de 22\% dos itens exigidos para as Boas Práticas de Armazenamento não foi cumprido, evidenciando a existência de problemas de armazenamento nos municípios.

A falta de infraestrutura adequada, a má gestão de estoque - aquisição acima ou abaixo da demanda - juntamente com as más condições de armazenamento, são os principais fatores que determinam o desperdício de recursos públicos destinados à aquisição de medicamentos.

Esse fato ficou ratificado nos relatos dos auditores da CGU, quando encontraram "medicamentos com prazos de validade expirados" em 10,9\% dos municípios paraibanos, percentual considerado elevado e agravado pelo registro da falta de medicamentos para atender à população. Isso sugere ineficiência nas etapas de seleção, programação, aquisição, controle e a inobservância das Boas Práticas de Armazenamento, bem como a ausência de recursos humanos capacitados para o desempenho das referidas funções.

A chance de um paciente receber um medicamento nessas condições o expõe a riscos de agravos à saúde, pois o uso de medicamento vencido pode tornar o tratamento inseguro, impróprio, ineficaz ou até mesmo nocivo à saúde (De Bernardi, Bieberbach e Thomé, 2006). 


\section{Componente - dispensação}

Em 34,5\% dos municípios, foi constatada a "falta de controle na dispensação dos medicamentos". Evidencia-se, portanto, que não se tem controle da quantidade de saída, do destino, se o medicamento realmente foi dispensado e, consequentemente, não se tem conhecimento da demanda de medicamentos não atendida, ou seja, da demanda reprimida. Ademais, em posição primordial está o bem-estar do paciente que necessita da orientação farmacêutica quanto ao uso adequado do medicamento. É justamente no ato da dispensação em que ocorre o contato do usuário com o farmacêutico, antes de iniciar o seu tratamento (Marin et al., 2003).

Assim como as demais ações desenvolvidas na farmácia, a dispensação precisa ser documentada, de preferência em sistema informatizado, contendo todas as informações sobre o paciente e a farmacoterapia indicada. $\mathrm{O}$ registro desses dados auxilia no acompanhamento do serviço de dispensação, assim como serve para subsidiar as intervenções que se façam necessárias, bem como o planejamento de ações de programas de saúde - itens esses que podem ser empregados como indicadores de qualidade dos serviços e resultados em saúde (Brasil, 2009).

Segundo a OMS, até $50 \%$ dos medicamentos são prescritos e/ou dispensados inadequadamente, e $50 \%$ são usados incorretamente pelos pacientes (WHO, 2004). Portanto, é de suma importância que o usuário obtenha uma prescrição adequada e uma orientação farmacêutica no ato de dispensação dos medicamentos, para resguardar a sua saúde. Assim, o ato de dispensação deve assegurar que o medicamento seja entregue ao paciente certo, na dose prescrita, na quantidade adequada, e que sejam fornecidas as informações suficientes para o uso correto (Opas, 1993).

\section{Componente - governança}

Dentre as categorias analisadas que constituem o componente governança, observou-se que a "ausência do profissional farmacêutico" foi constatada em apenas 3,6\% dos municípios estudados, resultado surpreendentemente positivo, a partir do conhecimento da realidade do estado. Um eventual falso positivo pode ter sido ocasionado pela forma como a pergunta foi feita, pois sabe-se da prática de alguns municípios de registrar a existência do profissional sem que este preste trabalho efetivamente na assistência farmacêutica ou pelo fato de, por vezes, esses profissionais desenvolverem suas atividades nos laboratórios de análises clínicas.

Assumido como verdadeiro, o resultado encontrado é melhor do que a situação evidenciada por Naves e Silver (2005) no Distrito Federal, onde somente dois Centros de Saúde, dentre os 15 estudados, possuíam farmacêuticos. É também melhor que os achados de Bruns (2013) no município de João Pessoa, capital do estado estudado, onde apenas duas Unidades de Saúde da Família, das 32 pesquisadas, contavam com o profissional farmacêutico de forma permanente.

Cabe assinalar que a ausência do farmacêutico é um dos dificultadores da implementação e da prestação de uma assistência farmacêutica de qualidade ao cidadão, além de configurar descumprimento da Lei no 5.991 , de 1973, que determina a obrigatoriedade da presença do farmacêutico em locais de dispensação de medicamentos. 
Adicionalmente, o Ministério da Saúde e vários autores vêm reforçando a importância da inserção plena da assistência farmacêutica na atenção primária de saúde, bem como a inclusão e a integração do profissional farmacêutico à equipe de saúde (Brasil, 2008; Oliveira, Bermudez e Osorio-de-Castro, 2007).

Outra categoria que merece atenção diz respeito aos "desvios de recursos e fraudes do programa de assistência farmacêutica", evidenciados em 38,2\% dos municípios estudados, configurando uma situação grave diante da escassez de recursos destinados à saúde, e trazendo fortes indícios referentes à malversação de recursos públicos. Esses dados coincidem com os relatados por Vieira (2008), que aponta, a partir de sua análise das regiões brasileiras, que os maiores percentuais de fraudes e desvios de recursos do programa de Assistência Farmacêutica foram detectados em municípios das regiões Nordeste (9\%) e Norte (6,8\%).

Adicionalmente, cabe assinalar que a "falta de comprovação dos gastos com os recursos da assistência farmacêutica" foi observada em 5,4\% dos municípios paraibanos, situação inadmissível, visto que a gestão da "coisa pública" requer que cada gestor apresente documentação comprobatória da utilização dos recursos sob a sua responsabilidade, atendendo aos princípios éticos, morais e legais.

Cabe ressaltar que um dos fatores que dificulta a efetivação das ações sistemáticas da assistência farmacêutica é a "falta de contrapartida da Secretaria Estadual de Saúde" para o Programa Farmácia Básica, constatada em 20\% dos municípios paraibanos, bem como a "falta de contrapartida da Secretaria Municipal de Saúde" para o referido Programa, em 9,1\% dos municípios auditados.

Verifica-se, portanto, que tanto o Estado como os municípios não vêm assumindo de forma sistemática as contrapartidas sob sua responsabilidade, conforme determina legislação vigente. Isso promove um descumprimento das normas legais, e traz prejuízos e transtornos para o sistema de saúde e para a população assistida.

Outro problema observado foi a "inexistência de um Plano Municipal de Assistência Farmacêutica Básica” em 12,7\% dos municípios, evidenciando, portanto, a falta de uma programação das necessidades decorrentes do perfil nosológico da população (Brasil, 1998), que visa assegurar o suprimento dos medicamentos destinados à atenção básica à saúde da população, integrando sua programação à do Estado, com foco no abastecimento permanente e oportuno, bem como o não cumprimento da orientação expressa na Portaria $n^{\circ} 2.084$, de 2005, em seu art. $8^{\circ}, \S 2^{\circ}$, quanto à elaboração e ao envio para o Estado do Plano Municipal de Assistência Farmacêutica.

\section{Componente - resultado}

É importante destacar que, em 27,3\% dos municípios, foi constatada a "falta de medicamentos essenciais", que fazem parte das listas oficiais pactuadas, de estoque mínimo e obrigatório. Segundo Rohde e colaboradores (2008) e Walley e colaboradores (2008), os medicamentos essenciais têm um papel importante e fundamental no desempenho da atenção primária à saúde. Por conseguinte, o referido resultado demonstra a falta de acesso dos usuários aos me- 
dicamentos. Isso representará a não resolutividade do seu problema de saúde, acarretando, portanto, prejuízos efetivos, não só na melhoria da qualidade de vida desses usuários, como na credibilidade do serviço prestado. Isso, muitas vezes, resulta em maiores complicações para o paciente e na necessidade de um nível mais complexo de atenção e, consequentemente, de custos mais elevados para o SUS, para a sociedade.

Este quadro evidencia o descumprimento, por parte dos municípios, da sua responsabilidade quanto à oferta dos medicamentos básicos assegurada pela legislação vigente, ferindo, portanto, o que preconiza a Constituição Federal de 1988 e o disposto na Lei no 8.080, de 1990, e no 12.401 , de 2011, que asseguram a assistência farmacêutica como direito do cidadão.

Estudos recentes sinalizam que boa parte dos medicamentos solicitados judicialmente faz parte das listas oficiais do SUS, o que demonstra claramente problemas de gestão na assistência farmacêutica da rede de serviços públicos (Chieffi e Barata, 2009; Marques e Dallari, 2007; Messeder, Osorio-de-Castro e Luiza, 2005; Pepe et al., 2010; Romero, 2008).

Contudo, cabe assinalar que esse quadro encontrado pelos auditores da CGU em municípios paraibanos é semelhante ao referenciado no trabalho de Oliveira, Assis e Barboni (2010), em que os referidos autores concluíram que existem baixa disponibilidade e descontinuidade da oferta de medicamentos essenciais em muitos municípios brasileiros; que a dispensação é realizada por trabalhadores sem qualificação; que a qualidade dos medicamentos é comprometida pelas condições inadequadas de armazenamento e que existem problemas relacionados ao acesso dos usuários à farmacoterapia.

Traçam-se, aqui, algumas comparações dos resultados do presente estudo com os achados de Vieira (2008). Salientamos que não há a apreensão de um caráter absoluto ou independente, considerando-as, entretanto, importantes em caráter relativo (tabela 3).

Tabela 3

Comparação dos resultados obtidos em municípios paraibanos com os dados encontrados na região Nordeste e os nacionais, de acordo com Vieira (2008)

\begin{tabular}{|c|c|c|c|}
\hline Categorias & Paraíba & Nordeste & Brasil \\
\hline Controle de estoque deficiente/inexistente de medicamentos & $52,7 \%$ & $68,1 \%$ & $71,4 \%$ \\
\hline Inobservância das normas de aquisição de medicamentos & $52,7 \%$ & $27,1 \%$ & $19,4 \%$ \\
\hline Desvio de recursos e/ou fraudes do Programa de Assistência Farmacêutica & $38,2 \%$ & $9,0 \%$ & $4,7 \%$ \\
\hline Falta de medicamentos & $27,3 \%$ & $30,3 \%$ & $24,1 \%$ \\
\hline Falta de contrapartida da SES & $20,0 \%$ & $13,8 \%$ & $13,6 \%$ \\
\hline Condições inadequadas de armazenamento & $20,0 \%$ & $43,1 \%$ & $39,0 \%$ \\
\hline $\begin{array}{l}\text { Aquisição de medicamentos com preço superior ao do banco de preços } \\
\text { em saúde (BPS) }\end{array}$ & $16,4 \%$ & $3,7 \%$ & $6,7 \%$ \\
\hline Medicamentos com validade expirada & $10,9 \%$ & $12,8 \%$ & $13,2 \%$ \\
\hline Falta de contrapartida da SMS & $9,1 \%$ & $7,4 \%$ & $8,4 \%$ \\
\hline Falta de comprovação dos gastos com os recursos da AF & $5,4 \%$ & $14,4 \%$ & $11,7 \%$ \\
\hline Ausência do Farmacêutico & $3,6 \%$ & $5,9 \%$ & $8,5 \%$ \\
\hline
\end{tabular}

Fonte: Elaboração própria, com base nos relatórios de fiscalização da Controladoria-Geral da União (CGU), disponíveis no endereço: $<$ www.cgu.gov.br/sorteios/index2.asp> e adaptados de Vieira (2008). 
Alguns dos resultados encontrados em municípios paraibanos mostram-se melhores, quando comparados com a região Nordeste e em nível nacional. Cita-se, como exemplo, a deficiência ou inexistência do controle de estoque de medicamentos, sendo constatados 52,7\%, $68,1 \%$ e $71,4 \%$, respectivamente, e as condições inadequadas de armazenamento, observadas em 20\% dos municípios paraibanos, em 43,1\% na região Nordeste e 39\% no Brasil.

Por outro lado, os achados apontam para algumas categorias que apresentaram resultados desfavoráveis em relação aos municípios paraibanos auditados. Os resultados correspondem aos percentuais referidos aos municípios paraibanos, ao Nordeste e ao Brasil, respectivamente. As categorias são: inobservância das normas de aquisição de medicamentos, que demonstrou 52,7\%, 27,1\% e 19,4\%; desvio de recursos e fraudes no Programa de Assistência Farmacêutica Básica, que apontou 38,2\%, 9\% e 4,7\%; aquisição de medicamentos com preço superior ao do Banco de Preços em Saúde, que foi identificada em 16,4\%, 3,7\% e 6,7\%; e a falta de contrapartida da Secretaria Estadual de Saúde, que representou 20\%, 13,8\% e $13,6 \%$.

Outros resultados encontrados são bastante semelhantes entre a Paraíba, o Nordeste e o Brasil, a exemplo daqueles relacionados à falta de medicamentos, aos medicamentos vencidos e à falta de contrapartida da Secretaria Municipal de Saúde.

Segundo Vieira (2008:91), "não é possível falar em efetividade da atenção à saúde sem que assistência farmacêutica cumpra o seu papel com qualidade".

\section{Considerações finais}

Considerado um dos estados mais pobres da nação, a Paraíba, consequentemente, possui escassos recursos destinados à saúde.

Foram evidenciados importantes problemas de gestão no período estudado, muitos deles evitáveis pelo simples respeito às normas existentes na legislação brasileira. Assim, de acordo com os resultados encontrados nos relatórios da CGU, podemos inferir que ainda há um longo percurso a ser construído para a efetivação e qualificação dos serviços farmacêuticos. Para tanto, é imprescindível uma conduta das três esferas de governo, no sentido de se profissionalizar a gestão pública, evitar toda e qualquer perda de recursos, tanto pela ineficiência administrativa, quanto pelos desvios e fraudes na condução da Política de Assistência Farmacêutica.

Dessa forma, a população poderá ser contemplada com o que lhe é assegurado por direito na legislação vigente: acesso aos medicamentos - que, segundo definição de Peters e colaboradores (2008:2), é "o uso oportuno de serviços de acordo com as necessidades" — e uma assistência farmacêutica de qualidade.

Cabe registrar que este trabalho não é específico para avaliar a qualidade da assistência farmacêutica municipal, mas sim para analisar a aplicação dos recursos federais, no caso, destinados à Assistência Farmacêutica Básica nos municípios. Apesar dessa limitação, sugerese que os resultados encontrados se mostram como uma alternativa factível e útil para um 
diagnóstico de como se encontra a assistência farmacêutica nos diversos municípios e qual a sua tendência.

A utilização desse tipo de dados secundários envolve baixo custo, e seu potencial de informações sobre a assistência farmacêutica é relevante, devendo, assim, ser difundido para que possa contribuir no sentido de diagnosticar possíveis problemas e fornecer subsídios às gestões federal, estadual e municipal, para o desenvolvimento de ações preventivas e corretivas, buscando a implementação das Políticas de Medicamentos e de Assistência Farmacêutica, para a qualificação dos serviços farmacêuticos nos municípios brasileiros.

Cabe assinalar que a base de informações se mostrou adequada para a análise pretendida. Contudo, estudos adicionais devem ser realizados para ampliar e aprofundar os achados.

A situação da assistência farmacêutica em municípios do estado da Paraíba, guardadas as devidas proporções, apresenta problemas e dificuldades semelhantes à realidade nacional, constatados nas referidas pesquisas. Os problemas encontrados quanto à assistência farmacêutica nos municípios do estado da Paraíba parecem, portanto, refletir a realidade dominante nos âmbitos do Nordeste e do Brasil. É fundamental que a utilização dos recursos públicos seja otimizada, para que se possa atender à elevada demanda de medicamentos, uma vez que grande parte da população tem reduzido poder aquisitivo e se encontra impossibilitada de obter esses ativos fora do sistema público de saúde.

\section{Referências}

ALI, Gamal K. M. How to establish a successful revolving drug fund: the experience of Khartoum state in the Sudan. Bulletin of the World Health Organization, v. 87, n. 2, p. 139-142, fev. 2009.

BANCO MUNDIAL. Governança no Sistema Único de Saúde (SUS) do Brasil: melhorando a qualidade do gasto público e gestão de recursos. Relatório № 36601- BR. Washington, DC: Banco Mundial, 2007. Disponível em: <http://siteresources.worldbank.org/brazilinporextn/resources/38171661185895645304/4044168-1186326902607/19governancasusport.pdf $>$. Acesso em: 7 nov. 2013.

BENEVIDES, Carolina; MENEZES, Maiá. Sobrepreço na compra de remédios, um mal nacional. $O$ Globo, Rio de Janeiro, 30 jan. 2011. Primeiro Caderno, p. 9.

BIGDELI, Maryam et al. Access to medicines from a health system perspective. Health policy and planning, v. 28, n. 7, p. 692-704, 2012.

BOING, Alexandra C.; BERTOLDI, Andréa D.; PERES, Karen G. Desigualdades socioeconômicas nos gastos e comprometimento da renda com medicamentos no Sul do Brasil. Revista de Saúde Pública, v. 45, n. 5, p. 897-905, maio 2011.

BRASIL. Conselho Nacional de Saúde. Resolução ㄲo 338, de 6 de maio de 2004. Aprova a Política Nacional de Assistência Farmacêutica. Diário Oficial da União, Brasília, 20 maio 2004. Seção 1, p. 52. 
BRASIL. Constituição (1988). Constituição da República Federativa do Brasil: texto constitucional promulgado em 5 de outubro de 1988, com as alterações adotadas pelas emendas Constitucionais no 1/1992 a 64/2010, pelo Decreto legislativo nํ 186/2008 e pelas emendas Constitucionais de Revisão no 1 a 6/1994. 32. ed. Brasília: Câmara dos Deputados, edições Câmara, 2010a.

BRASIL. Controladoria-Geral da União. Portaria no 247 CGU, 20 de junho de 2003. Institui, em caráter permanente, o mecanismo do sorteio público para definição das unidades municipais onde será objeto de fiscalização a aplicação de recursos públicos federais. Diário Oficial da União, Brasília, 20 jun. 2003. Seção 1, p. 13.

BRASIL. Ministério da Saúde. Assistência farmacêutica na atenção básica: instruções técnicas para a sua organização. Brasília, DF: Ministério da Saúde, 2006. Disponível em: <www.sbpt.org.br/ downloads/arquivos/COM_DPOC/IRTB2012_doc01.pdf>. Acesso em: 17 mar. 2014.

BRASIL. Ministério da Saúde. Portaria no 154, de 24 de janeiro de 2008. Cria os Núcleos de Apoio à Saúde da Família — Nasf. Diário Oficial da União, Brasília, 25 jan. 2008. Seção 1, p. 47.

BRASIL. Ministério da Saúde. Portaria no 176, de 08 de março de 1999. Estabelece critérios e requisitos para a qualificação dos municípios e estados ao Incentivo a Assistência Farmacêutica Básica e define valores a serem transferidos. Diário Oficial da União, Brasília, 11 mar. 1999, Seção 1, p. 22.

BRASIL. Ministério da Saúde. Portaria n 2.084, de 28 de outubro de 2005. Estabelece normas, responsabilidades e recursos a serem aplicados no financiamento da Assistência Farmacêutica na Atenção Básica e define o Elenco Mínimo Obrigatório de Medicamentos. Diário Oficial da União, Brasília, 28 out. 2005.

BRASIL. Ministério da Saúde. Portaria no 3.916, de 30 de outubro de 1998. Aprova a Política Nacional de Medicamentos. Diário Oficial da União, Brasília, 10 nov. 1998. Seção 1, p. 18.

BRASIL. Ministério da Saúde. Portaria no 4.217, de 28 de dezembro de 2010. Aprova as normas de financiamento e execução do Componente Básico da Assistência Farmacêutica. Diário Oficial da União, Brasília, 28 dez. 2010b. Seção 1, p. 72.

BRASIL. Ministério da Saúde. Secretaria de Ciência, Tecnologia e Insumos Estratégicos. Departamento de Assistência Farmacêutica e Insumos Estratégicos. Diretrizes para estruturação de farmácias no âmbito do Sistema Único de Saúde. Brasília: Ministério da Saúde, 2009.

BRASIL. Ministério da Saúde. Secretaria de Políticas de Saúde. Departamento de Atenção Básica. Incentivo à Assistência Farmacêutica Básica: o que é e como funciona. Elaborada por Geraldo Luchesi et al. Brasília: Ministério da Saúde, 2001.

BRASIL. Presidência da República. Lei no 8.080, de 19 de setembro de 1990. Dispõe sobre as condições para promoção, proteção e recuperação da saúde, a organização e o funcionamento dos serviços correspondentes e dá outras providências. Diário Oficial da União, Brasília, 20 set. 1990. Seção 1, p. 18055. 
BRASIL. Presidência da República. Lei no 8.666, de 21 de junho de 1993. Regulamenta o art. 37, inciso XXI, da Constituição Federal, institui normas para licitações e contratos da Administração Pública e dá outras providências. Diário Oficial da União, Brasília, 22 jun. 1993. Seção 1, p. 8269.

BRASIL. Presidência da República. Lei no 10.520, de 17 de julho de 2002. Institui, no âmbito da União, estados, Distrito Federal e municípios, nos termos do art. 37, inciso XXI, da Constituição Federal, modalidade de licitação denominada pregão, para aquisição de bens e serviços comuns, e dá outras providências. Diário Oficial da União, Brasília, 18 jul. 2002. Seção 1, p. 1

BRASIL. Presidência da República. Lei no 12.401, de 28 de abril de 2011. Altera a Lei no 8.080, de 19 de setembro de 1990, para dispor sobre a assistência terapêutica e a incorporação de tecnologia em saúde no âmbito do Sistema Único de Saúde - SUS. Diário Oficial da União, Brasília, 29 abr. 2011. Seção 1, p. 1.

BRUNS, Suelma de Fátima. Política de assistência farmacêutica no município de João Pessoa-PB: contexto, desafios e perspectivas. 2013. Tese (doutorado em saúde pública) — Escola Nacional de Saúde Pública Sergio Arouca, Rio de Janeiro, 2013.

BRUNS, Suelma de Fátima; LUIZA, Vera L.; OLIVEIRA, Egléubia A. Judicialização da assistência farmacêutica: dois pesos e uma medida. In: OLIVEIRA, Maria Helena de et al. (Org.). Direito e saúde: cidadania e ética na construção de sujeitos sanitários. Maceió: Edufal, 2013. p. 366-390.

CAMERON, Alexandra et al. Medicine prices, availability, and affordability in 36 developing and middle-income countries: a secondary analysis. The Lancet, v. 373, n. 9659, p. 240-249, 23 jan. 2009.

CARASSO, Barbara S. et al. Availability of essential medicines in Ethiopia: an efficiency-equity trade-off? Tropical Medicine and International Health, v. 14, n. 11, p. 1394-1400, nov. 2009.

CHIAVENATO, Idalberto. Introdução à teoria geral da administração: uma visão abrangente da moderna administração das organizações. Rio de Janeiro: Elsevier, 2003.

CHIEFFI, Ana L.; BARATA, Rita B. Judicialização da política pública de assistência farmacêutica e equidade. Cadernos de Saúde Pública, v. 25, n. 8, p. 1839-1849, ago. 2009.

CONSELHO REGIONAL DE MEDICINA DO ESTADO DE SÃO PAULO. Conselho Regional de Farmácia do Estado de São Paulo. Instituto Brasileiro de Defesa do Consumidor. Medicamento um direito essencial. São Paulo, 2006. Disponível em: <www.dhnet.org.br/dados/cartilhas/a_pdf/114_cartilha_direito_medicamentos.pdf $>$. Acesso em: 9 nov. 2013.

DE BERNARDI, Carmen L. B.; BIEBERBACH, Emily W.; THOMÉ, Henrique I. Avaliação da assistência farmacêutica básica nos municípios de abrangência da $17^{\text {a }}$ Coordenadoria Regional de Saúde do Rio Grande do Sul. Saúde e Sociedade, v. 15, n. 1, p. 73-83, abr. 2006.

FLEURY, Paulo F.; WANKE, Peter; FIGUEIREDO, Kleber. Logística empresarial: a perspectiva brasileira. São Paulo: Atlas, 2000.

INSTITUTO BRASILEIRO DE GEOGRAFIA E ESTATÍSTICA (IBGE). Contagem da população 2010. Disponível em: <www.ibge.gov.br/home/estatistica/populacao/censo2010/primeiros_resultados/ populacao_por_municipio.shtm>. Acesso em: 6 maio 2011. 
MARIN, Nelly et al. (Org.). Assistência farmacêutica para gerentes municipais. Rio de Janeiro: Opas/ OMS, 2003.

MARQUES, Silvia B.; DALLARI, Sueli G. Garantia do direito social à assistência farmacêutica no estado de São Paulo. Revista de Saúde Pública, v. 41, n. 1, p. 101-107, 2007.

MESSEDER, Ana M.; OSORIO-DE-CASTRO, Claudia G. S.; LUIZA, Vera L. Mandados judiciais como ferramenta para garantia do acesso a medicamentos no setor público: a experiência do estado do Rio de Janeiro, Brasil. Cadernos de Saúde Pública, v. 21, n. 2, p. 525-534, 2005.

NAVES, Janeth de O. S.; SILVER, Lynn D. Evaluation of pharmaceutical assistance in public primary care in Brasília, Brazil. Revista de Saúde Pública, v. 39, n. 2, p. 223-230, abr. 2005.

OLIVEIRA, Luciane C. F. de; ASSIS, Marluce M. A.; BARBONI, André R. Assistência farmacêutica no Sistema Único de Saúde: da política nacional de medicamentos à Atenção Básica à Saúde. Ciência \& Saúde Coletiva, v. 15, n. 3, p. 3561-3567, nov. 2010.

OLIVEIRA, Maria A.; BERMUDEZ, Jorge A. Z.; OSORIO-DE-CASTRO, Claudia G. S. Assistência farmacêutica e acesso a medicamentos. Rio de Janeiro: Fiocruz, 2007.

OPAS. Organização Pan-Americana da Saúde/Organização Mundial da Saúde. Avaliação da assistência farmacêutica no Brasil. Brasília: Organização Pan-Americana da Saúde, 2005.

OPAS. Organização Pan-Americana de Salud. El papel del farmacéutico en el sistema de atención de salud. Informe de La Reunión de la OMS Tokio, Japón, 31 de agosto al 3 de septiembre de 1993. Tokio: Organização Pan-Americana de Salud, 1993.

PEPE, Vera L. E. et al. Caracterização de demandas judiciais de fornecimento de medicamentos "essenciais" no estado do Rio de Janeiro, Brasil. Cadernos de Saúde Pública, v. 26, n. 3, p. 461-471, mar. 2010.

PETERS, David H. et al. Poverty and access to health care in developing countries. Annals of the New York Academy of Sciences, v. 1136, n. 1, p. 161-171, 2008.

ROHDE, Jon et al. 30 years after Alma-Ata: has primary health care worked in countries? The Lancet, v. 372, n. 9642 , p. $950-961,13$ set. 2008.

ROMERO, Luiz C. Judicialização das políticas de assistência farmacêutica: o caso do Distrito Federal. Texto para Discussão, n. 41, p. 48, maio 2008.

SANTOS, Lenir. Articulação federativa e contrato de ação pública no SUS. Blog Direito Sanitário: Saúde e Cidadania. 15 jun. 2011. Disponível em: <http://blogs.bvsalud.org/ds/2011/06/15/ articulacao-federativa-e-contrato-de-acao-publica-no-sus/>. Acesso em: 11 fev. 2013.

SIQUEIRA, Fábio; GAUDARD, Ana M. Y. S. Acesso aos serviços de farmácia em atenção básica na SES-DF. Comunicação em Ciências da Saúde, v. 20, n. 3, p. 211-217, 2009.

VASCONCELOS, Agdo. O princípio da eficiência na gestão pública. Governo e Política, 16 fev. 2009. Disponível em: <www.webartigos.com/artigos/o-principio-da-eficiencia-na-gestaopublica/14519/>. Acesso em: 5 nov. 2013. 
VAZ, José C.; LOTTA, G. S. A contribuição da logística integrada às decisões de gestão das políticas públicas no Brasil. Rev. Adm. Pública, v. 45, n. 1, p. 107-139, jan./fev. 2011.

VIEIRA, Fabiola S. Assistência farmacêutica no sistema público de saúde no Brasil. Revista Panamericana de Salud Pública, v. 27, n. 2, p. 149-156, 2010.

VIEIRA, Fabiola S. Gasto do Ministério da Saúde com medicamentos: tendência dos programas de 2002 a 2007. Revista de Saúde Pública, v. 43, n. 4, p. 674-681, 2009.

VIEIRA, Fabiola S. Qualificação dos serviços farmacêuticos no Brasil: aspectos inconclusos da agenda do Sistema Único de Saúde. Revista Panamericana de Salud Pública, v. 24, n. 2, p. 91-100, ago. 2008.

WALLEY, John et al. Primary health care: making Alma-Ata a reality. The Lancet, v. 372, n. 9642, p. 1001-1007, 19 set. 2008.

WHO, World Health Organization. WHO medicines strategy — countries at the core: 2004-2007. Geneva: World Health Organization, 2004.

Suelma de Fátima Bruns é doutora em saúde pública, mestre em administração de empresas e professora adjunta do Departamento de Ciências Farmacêuticas da Universidade Federal da Paraíba (DCF/UFPB). E-mail: suelmadefatima@yahoo.com.br.

Vera Lucia Luiza é doutora em saúde pública, mestre em farmácia hospitalar e pesquisadora titular do Núcleo de Assistência Farmacêutica, Escola Nacional de Saúde Pública Sergio Arouca, Fundação Oswaldo Cruz (NAF/ENSP/Fiocruz). E-mail: vera@ensp.fiocruz.br.

Egléubia Andrade de Oliveira é doutora em saúde pública e pesquisadora do Instituto de Estudos em Saúde Coletiva do Departamento de Bioética da Universidade Federal do Rio de Janeiro (Iesc/UFRJ). E-mail: biaol@ensp.fiocruz.br. 
